MATHEMATICS OF GRAVITATION

PART II, GRAVITATIONAL WAVE DETECTION

BANACH CENTER PUBLICATIONS, VOLUME 41

INSTITUTE OF MATHEMATICS

POLISH ACADEMY OF SCIENCES

WARSZAWA 1997

\title{
GRAVITATIONAL WAVEFORMS FROM SPINNING OBJECTS
}

\author{
THEOCHARIS A. APOSTOLATOS \\ Max-Planck-Society, Research Unit "Theory of Gravitation" \\ at the Friedrich-Schiller-University, D-07743 Jena, Germany \\ E-mail: taa@gravi.physik.uni-jena.de
}

\begin{abstract}
According to general relativity, a binary consisting of spinning bodies will precess due to spin-orbit and spin-spin coupling. The corresponding modulation of its gravitational waves might be a serious problem for detecting such waves with simple post-Newtonian templates. A new family of templates that takes into account the complications arising from the orbital precession is proposed and its application and performance are discussed.
\end{abstract}

1. Introduction. Inspiraling compact binaries are the most promising source of gravitational waves for a worldwide network of ground-based laser-interferometer detectors that is already under construction. If at least one of the compact bodies is rapidly spinning, their spin-orbit and spin-spin coupling will make their orbit to precess and accordingly their gravitational waves will be modulated both in amplitude and in phase. But strongly modulated signal waveforms require much more complicated templates than the simple post-Newtonian ones to be correlated with, in order to be detected. On the other hand, these modulated signals require a huge number of search templates to be used for detection purposes.

In section 2, I will describe the equations governing the precession of the orbital plane and I will discuss the corresponding solution for some simple but interesting case, namely the case where only one of the bodies is rapidly spinning (see Ref. [1,7]); the general case is briefly discussed. The consequences of precession on the shape of gravitational waves will be presented as well. In section 3, I will explain the problems that precession might cause in our efforts to detect the gravitational waves from such a binary, and I will present a new extended family of templates that exhibits a much better detection performance than the simple post-Newtonian templates. The problem though is that this new family is so rich in templates that makes data analysis in real time rather impossible. Nevertheless, it could be useful in a hierarchical search,

1991 Mathematics Subject Classification: Primary 46C20; Secondary 32G81.

The paper is in final form and no version of it will be published elsewhere. 
where one could look for spin-related complications of the signals in an after-detection search.

Throughout I use units where $G=c=1$.

2. Equations of precession and wave modulation. After specializing to circular orbits and after averaging over one orbit, the equations describing the orbital precession of a binary with masses $M_{1}, M_{2}$ and spins $\vec{S}_{1}, \vec{S}_{2}$ take the following form, accurate through post $^{2}$-Newtonian order [1]:

$$
\begin{gathered}
\dot{\vec{S}}_{1}=\frac{1}{r^{3}}\left[\frac{4 M_{1}+3 M_{2}}{2 M_{1}} \vec{L}+\frac{1}{2} \vec{S}_{2}-\frac{3}{2}\left(\vec{S}_{2} \cdot \hat{L}\right) \hat{L}\right] \times \vec{S}_{1}, \\
\dot{\vec{S}}_{2}=\frac{1}{r^{3}}\left[\frac{4 M_{2}+3 M_{1}}{2 M_{2}} \vec{L}+\frac{1}{2} \vec{S}_{1}-\frac{3}{2}\left(\vec{S}_{1} \cdot \hat{L}\right) \hat{L}\right] \times \vec{S}_{2}, \\
\dot{\vec{L}}=-\dot{\vec{S}}_{1}-\dot{\vec{S}}_{2}-\frac{32}{5} \frac{\mu^{2}}{r}\left(\frac{M}{r}\right)^{5 / 2} \hat{L} .
\end{gathered}
$$

Here an overdot represents 'd/dt', $M$ and $\mu$ are the total and reduced masses respectively, $\hat{L}$ is the unit vector along the orbital angular momentum $\vec{L}$, and $r$ is the separation distance between the two bodies. By solving the Eqs. (1)-(3) one can follow the evolution of $\hat{L}$, that is, the evolution of the precession of the orbital plane.

A very interesting case where Eqs. (1)-(3) can be solved analytically is the case where one of the two bodies has vanishing spin (e.g., a binary consisting of a rapidly spinning black hole and a non-rotating neutron star). One then can show that $\hat{L}$ moves on a tight outward (or inward - depending on the misalignment angle between $\vec{L}$ and $\vec{S}$ ) spiral trail around the total angular momentum of the binary $\vec{J}$, the direction of which remains almost fixed, as the orbital angular momentum gets radiated away due to gravitational wave emission. This is what we have called the simple precession [1]. Another very interesting, but rather rare, case is when $\vec{L}$ and $\vec{S}$ (again one of the spins is assumed to be zero) are almost anti-aligned. At some epoch $\vec{L}$ and $\vec{S}$ become comparable in magnitude and they almost cancel each other; the binary then loses its gyroscopic stability and tumbles in space. Unfortunately no analytic solution has been found for this, so called, transitional precession.

According to our numerical investigations (see Ref. [1]), binaries with arbitrary masses and spins undergo ragged versions of the simple precession described in the previous paragraph. Finally,two more special cases can be approximately solved analytically: (i) when one of the spins is much smaller than the other and therefore it can be treated perturbatively, leading to an epicyclic motion on top of the single-spin precession [1], and (ii) when the two bodies have equal masses and spins (e.g., two neutron stars with the same spinning rate); see Ref. [4].

Because of the spin-induced orbital precession, the geometry of the binary with respect to a fixed detector on Earth is continuously changing, leading to a modulated signal of the form:

$$
h(t)=A_{N}(t) \times \mathrm{AM}(t) \cos \left(2 \int \Omega(t) d t+\mathrm{PM}(t)\right),
$$


where $A_{N}(t)$ represents the non-modulated part of the Newtonian amplitude, $\Omega(t)$ is the orbital angular velocity of the binary, and $\operatorname{AM}(t)$ and $\operatorname{PM}(t)$ are the amplitude and phase modulation caused by precession. These modulation factors might evolve secularly, depending on the parameters and the initial geometry of the binary, but they mainly exhibit oscillatory behavior. The precession angle $\alpha$ evolves with frequency as $\propto f^{-2 / 3}$ to $f^{-1}$ depending on the relative sizes of $L$ and $S$ [1]. This also is the frequency dependence of $\operatorname{AM}(t)$ and $\operatorname{PM}(t)$. According to my analysis [2], $\operatorname{PM}(t)$ is potentially the most dangerous deforming factor; that was expected since it is the phase matching between the signal and the template that mainly determines the output of the correlation [5].

3. Consequences in detecting the modulated waves. For some special geometrical configurations of the binary with respect to the detector, the modulation of the waves is very deep; therefore, the simple post-Newtonian templates are very ineffective in detecting such gravitational waves. The signal-to-noise reduces dramatically because of using a template family none of whose members can accurately reproduce the modulated waveform. The situation is worse for binaries, for which the opening angle for the precession of their $\hat{L}$ is large (where the opening angle for the precession of $\hat{L}$ is the angle between $\hat{L}$ and the direction around which $\vec{L}$ precesses). To be more specific, one introduces a quantity (the so called Fitting Factor, $F F[2]$ ) which measures the reduction in signal-to-noise that results from using some family of templates, rather than the true signal. One then looks for the fraction of geometrical configurations of the binary that produce $F F$ 's above, say, 0.9. For a neutron star/neutron star binary with only one of its bodies rapidly spinning $\left(S_{i}=M_{i}^{2}\right)$, the waveform of which has been computed using the Newtonian quadrupole moment formalism, a family of Newtonian non-modulated templates produces $F F$ 's above 0.9 for almost all possible geometries and misalignment angles between $\vec{L}$ and $\vec{S}$, while for a $10 M_{\odot} / 1.4 M_{\odot}$ black hole/neutron star binary with only the black hole maximally spinning, $F F$ is below 0.9 for more than half of the geometries when the misalignment angle exceeds $30^{\circ}$. (The difference between the two types of binaries is due to the ratio of masses of the two bodies; in the NS/NS case the opening angle for the precession of $\vec{L}$ cannot be too large, whatever the misalignment angle is.) If, on the other hand, higher post-Newtonian effects (up to post ${ }^{1.5}$-Newtonian order) are added on the phase of the signal, and a post ${ }^{1.5}$-Newtonian family of templates with vanishing spin parameter is used for detecting the signal, then the NS/NS case will produce equally good results as before ( $F F$ above 0.9 for almost all possible geometries), but for the $\mathrm{BH} / \mathrm{NS}$ case the fraction of geometries of binaries with misalignment angles equal to $30^{\circ}$ which are producing $F F<0.9$ is now reduced to roughly $1 / 3$. The better performance of the post ${ }^{1.5}$-Newtonian templates is due to the larger flexibility of the post-Newtonian templates, compared to the Newtonian ones, in mimicing a more complicated waveform, like a precessionally modulated one; the post ${ }^{1.5}$-Newtonian templates have one more parameter to adjust than the Newtonian ones.

In order to improve even more the performance of the post ${ }^{1.5}$-Newtonian templates I have tried [3] to add on their phase an extra term of the form

$$
\psi_{1.5}^{\mathrm{NEW}}(f)=\psi_{1.5}^{\mathrm{OLD}}(f)+\mathcal{C} \cos \left(\delta+\mathcal{B} f^{-2 / 3}\right)
$$


where $\psi_{1.5}^{\mathrm{NEW}}(f)$ and $\psi_{1.5}^{\mathrm{OLD}}(f)$ are the phase functions of the new and the old templates respectively, and $\mathcal{C}, \delta, \mathcal{B}$ are the three extra parameters the proposed templates depend on. This special form of the term, which has been added on the phase of the old post ${ }^{1.5}$ Newtonian templates, is justified by the fact that the secular evolution of PM could be imitated quite well by appropriately adjusting the parameters of the post ${ }^{1.5}$-Newtonian templates [3], but then, it still remains the oscillatory evolution of the signal's phase modulation that cannot be corrected otherwise. The frequency dependence $\propto f^{-2 / 3}$ of the oscillatory extra term shown in Eq. (4) is chosen to coincide with the approximate frequency dependence of the precession but the specific power dependence is not crucial.

Of course, this extended family of templates cannot follow very accurately the evolution of a realistic modulated gravitational wave, but at least in the region of frequencies where the detectors are most sensitive, it can mimic such a wave quite satisfactorily. The price one has to pay then is that the inclusion of these 3 extra parameters, $\mathcal{C}, \delta, \mathcal{B}$, will skyrocket the number of templates to a formidable $10^{11}-10^{12}$; real-time data analysis is not possible with such a number of templates. These new templates, however, could be used at a more detailed after-detection stage to improve the signal-to-noise and to help acquiring more precise information about the parameters of the binary by offering some useful information about the spin of the bodies [6].

\section{References}

[1] T. A. Apostolatos, C. Cutler, G. J. Sussman, and K. S. Thorne, Spin-induced Orbital Precession and its Modulation of the Gravitational Waveforms from Merging Binaries, Phys. Rev. D 49, 6274 (1994).

[2] T. A. A postolatos, Search templates for gravitational waves from precessing, inspiraling binaries, Phys. Rev. D 52, 605 (1995).

[3] T. A. Apostolatos, Construction of a template family for the detection of gravitational waves from coalescing binaries, to be published in Phys. Rev. D.

[4] T. A. Apostolatos, The influence of spin-spin coupling on inspiraling compact binaries with $M_{1}=M_{2}$ and $S_{1}=S_{2}$, to be published in Phys. Rev. D.

[5] C. Cutler, T. A. Apostolatos, L. Bildsten, L. S. Finn, E. Flanagan, D. Kennefick, D. M. Markovic, A. Ori, E. Poisson, G. J. Sussman, and K. S. Thorne, The last three minutes: Issues in gravitational wave measurements of coalescing binaries, Phys. Rev. Lett. 70, 2984 (1993).

[6] C. Cutler and E. Flanagan, Gravitational waves from merging compact binaries: How accurately can one extract the binary's parameters from the inspiral waveform?, Phys. Rev. D 49, 2658 (1994).

[7] K. S. Thorne and J. B. Hartle, Laws of motion and precession for black holes and other bodies, Phys. Rev. D 31, 1815 (1985). 